\title{
Accidentes con material biológico en trabajadores de la salud. Palmira-Valle del Cauca. 2014-2016
}

\author{
Accidents with biological material in health workers. Palmira-Valle del Cauca. 2014-2016 \\ Acidentes com material biológico em trabalhadores da saúde, Palmira,Valle del Cauca \\ (2014-2016)
}

Mercedes Salcedo-Cifuentes*

Paula Andrea Arango-Correa**

John Jairo Medina-Murillo***

\begin{abstract}
Autor de correspondencia
* $\triangle$ Bacterióloga. Magister en Epidemiología y Doctor en Ciencias Biomédicas. Docente Universidad del Valle. Investigadora del grupo CALIMET. Correo: mercedes. salcedo@correounivalle.edu.co. (iD https:// orcid.org/0000-0002-9681-6893. Cali, Colombia.

** Enfermera. Magister en Salud Ocupacional de la Universidad Libre. Seccional Cali. Funcionaria de Clínica Palmira S.A. Correo: paulaandreaarangoc@hotmail.com. (iD https:// orcid.org/0000-0001-6260-0448. Palmira, Colombia.

*** Estadístico. Magister en Epidemiología de la Universidad del Valle. Funcionario de la Secretaria de Tránsito Municipal. Profesor Auxiliar de la Corporación Universitaria José Antonio Camacho. Correo: jhonjai24@ gmail.com. (D) https://orcid.org/0000-00030808-8716. Cali, Colombia.
\end{abstract}

Este es un artículo bajo la licencia CC BY

(https://creativecommons.org/

licenses/by/4.0/) @) (1)

\begin{abstract}
Resumen
Introducción: Los profesionales sanitarios están expuestos diariamente a diferentes riesgos biológicos generados por su ambiente laboral. Objetivo: Describir el perfil de los accidentes laborales por riesgo biológico en una institución de salud de segundo nivel de complejidad. Palmira-Valle del Cauca. 2014-2016. Materiales y Métodos: Estudio descriptivo, retrospectivo, realizado con 37 reportes. A las variables cuantitativas se les calcularon medidas de tendencia central y dispersión y a las cualitativas proporciones y razones. Para analizar la relación entre variables se usaron los Test del Chi cuadrado, Fisher o Medianas de acuerdo a la distribución de los datos, considerando un valor de $p \leq 0,05$ como significante. Resultados: La mayoría de los trabajadores accidentados fueron mujeres jóvenes, principalmente enfermeras auxiliares las cuales fueron lesionadas por elementos punzantes (agujas) durante los turnos matutinos y en los servicios de mayor demanda (urgencias y hospitalización). El $89 \%$ de los trabajadores expuestos al riesgo biológico no utilizaron materiales o equipos de seguridad al momento del accidente. Las causas frecuentes fueron la manipulación de agujas y los movimientos del paciente al realizar procedimientos asistenciales. Las manos fueron el área más afectada. Conclusiones: El elemento vulnerante, el sitio de la lesión, el servicio, la edad del trabajador, el turno y el cargo en la institución prestadora de servicios de salud, determinaron el perfil de los accidentes de trabajo, que registraron el mayor número de accidentes laborales con exposición a riesgo biológico; las causas más frecuentes se asociaron con la manipulación de elementos punzantes cuando la asistente atiende al paciente.
\end{abstract}

Palabras claves: Accidente de trabajo, exposición a agentes biológicos, factores de riesgo.

\section{Abstract}

Introduction: Sanitary professionals are exposed daily to different biological risks 
generated by their work environment Objective: Describe the profile of work accidents caused by biological risks in a secondary level health care institution. Palmira-Valle del Cauca. 20142016. Materials and Methods: Descriptive, retrospective study performed with 37 reports. For the quantitative variables, central tendency and dispersion were calculated, and for the qualitative variables, proportions and ratios. To analyze the relation between variables the Pearson's chisquared test, fisher or the median were used according to the distribution of data, considering a value of $p \leq 0,05$ as significant. Results: Most of the workers injured were young women, mainly auxiliary nurses which were injured by sharps (needles) during morning shifts and in services of greater demand (emergencies and hospitalization). $89 \%$ of the workers exposed to biological risk did not use safety materials or equipment at the moment of the accident. The frequent causes were needle manipulation and movements of the patient when performing assistant procedures. Hands were the most affected area. Conclusions: The object that caused the accident, the place of the injury, the service, the age of the worker, the shift and the charge in the health care service institution, determined the profile of the work accidents which registered the greatest number of work accidents with exposure to biological risks; the most frequent causes were associated to the manipulation of sharp objects when the assistant or nurse attends the patient.

Keywords: Work accident, exposure to biological agents, risk factors.

\section{Resumo}

Introdução: Os professionais sanitários estão expostos diariamente a diferentes riscos biológicos gerados por seu ambiente de trabalho. Objetivo: Descrever o perfil dos acidentes no trabalho por risco biológico numa instituição de saúde de segundo nível de complexidade, na cidade de Palmira, Valle del Cauca, Colômbia (2014-2016). Materiais e Métodos: Estudo descritivo, retrospectivo, realizado com 37 relatórios. Às variáveis quantitativas se lhes calcularam medidas de tendência central e dispersão e às qualitativas proporções e razões. Para analisar a relação entre variáveis se usaram os Teste do Qui-quadrado, Fisher ou Medianas de acordo à distribuição dos dados, considerando um valor de $\mathrm{p} \leq 0,05$ como significativo. Resultados: A maioria dos trabalhadores acidentados foram mulheres jovens, principalmente auxiliares de enfermagem as quais foram lesionadas por elementos perfurocortantes (agulhas) durante os turnos da manhã e nos serviços de maior demanda (emergências e hospitalização). O $89 \%$ dos trabalhadores expostos ao risco biológico não utilizaram materiais ou equipamentos de segurança ao momento do acidente. As causas frequentes foram a manipulação de agulhas e os movimentos do paciente ao realizar procedimentos de cuidados de saúde. As mãos foram a área mais afeitada. Conclusões: O elemento vulnerável, o local da lesão, o serviço, a idade do trabalhador, o turno e o cargo na instituição fornecedora de serviços de saúde; determinaram o perfil dos acidentes no trabalho que registraram o maior número de acidentes com exposição a risco biológico; as causas mais frequentes se associaram com a manipulação de elementos pontiagudos quando a auxiliar de enfermagem cuidava do paciente.

Palavras-chave: Acidente no trabalho, exposição a agentes biológicos, fatores de risco.

\section{Introducción}

Las lesiones por pinchazos con agujas son los accidentes de trabajo (AT) más comunes en la atención en servicios de salud y suponen un grave riesgo para los trabajadores. Las tasas de ocurrencia varían entre el 3,2 al 24,7 por cada 100 camas, según el nivel de complejidad de la institución hospitalaria y país (1).

Los AT con exposición a riesgo biológico no ocurren por el azar, son el resultado de una serie de factores que interactúan y potencia su ocurrencia, los cuales de acuerdo a su nivel de intervención pueden ser modificables y no modificables (1). Dentro de las principales grupos de factores asociadas a estos eventos se encuentran: factores de ingeniería, incluida la forma de los dispositivos afilados y dispositivos de barrera; factores organizativos, en los cuales se enmarca la existencia de suministros y políticas para la presentación de informes; por último, los factores comportamentales de los trabajadores de la salud, entre los que se incluyen: el uso indiscriminado de agujas, el re-encapsulado de las mismas y el poco uso o no de los elementos de bioseguridad (2). 
La Organización Mundial de la Salud (OMS) ha informado que la falta de disponibilidad de controles de ingeniería como agujas con dispositivos más seguros, el bajo número de personal frente a la demanda en los servicios y el fracaso en la sensibilización e inducción en el estricto cumplimiento de los protocolos de seguridad de cada procedimiento asistencial, parecen ser los factores que más contribuyen al riesgo (3). En este último factor, el Centro Internacional de Seguridad de los Trabajadores de la Salud en EE.UU y otros estudios $(2,4)$, han demostrado que los riesgos presentes antes, durante y después del procedimiento de inyectología son las causas que más prevalecen en los accidentes de trabajo en servicios de salud; le siguen en su orden punciones durante la extracción de sangre venosa, canalización de venas y la toma de muestras de sangre para el laboratorio clínico.

Masoumi et al. (5) y Cho et al. (6) han identificado además que el turno laboral, el número de horas trabajadas de manera consecutiva que conllevan al agotamiento físico del trabajador, el servicio donde se presentó el AT, la capacitación en diferentes temas de seguridad y salud laboral, la experiencia laboral, entre otros, son de igual forma factores que contribuyen a estos eventos los cuales involucran con mayor frecuencia al personal de enfermería.

Estudios nacionales que coincidan con estos hallazgos son pocos, por ejemplo, Montufar et al. (7) así como López et al. (8) coincidieron en señalar que el personal más afectado son los tecnólogos en enfermería y las lesiones percutáneas $(>75 \%)$ en las manos ( $>80 \%)$, son las características que definen el perfil ocupacional de los AT con exposición a riesgo biológico en los trabajadores de la salud. Además, las actividades o prácticas que conllevan a mayor riesgo son la preparación y administración de medicamentos, las suturas, la toma de muestras de sangre y el no uso de EPP. De otra parte, para varios expertos el reporte de los AT por exposición a riesgo biológico no refleja la verdadera prevalencia $(9,10)$. En un seguimiento activo, la frecuencia de exposición a pinchazos con agujas en el último mes fue del $36 \%$ y en los últimos tres meses de $37,6 \%$, resultados mucho más altos que las estadísticas reportadas en un año (11).

Un análisis integral de todas las causas lleva a concluir que los contextos de la ocurrencia de los accidentes de trabajo con material biológico varían según el nivel de complejidad (12), del recurso humano y financiero del cual disponen los diferentes tipos de establecimientos de salud. En este mismo sentido, la gestión de los programas de seguridad y salud laboral difiere significativamente. Los hospitales docentes tienden a ejercer prácticas de control de infecciones y gestión del proceso del accidente laboral de manera más efectiva, frente a los hospitales de las redes de salud pública, que a menudo son más pequeños, reciben menos incentivos financieros y tienen una respuesta poco oportuna frente a la gestión de estos procesos (13-15).

Los riesgos biológicos en las instituciones prestadoras de servicios de salud difieren en su magnitud e impacto, por lo cual definir los factores desencadenantes o contribuyentes al accidente laboral con material biológico es prioritario, para profundizar en el conocimiento de uno de los eventos más frecuentes en los trabajadores de la salud y al mismo tiempo para incentivar las acciones de mejora en el sistema de gestión de seguridad y salud laboral.

\section{Objetivos}

\section{Objetivo General}

Describir el perfil de los accidentes laborales con material biológico en los trabajadores de una institución de salud de segundo nivel de complejidad. Palmira-Valle del Cauca. 2014-2016.

\section{Objetivos Específicos}

- Identificar los factores que contribuyen a los accidentes laborales por riesgo biológico.

- Evaluar la relación entre el accidente de trabajo por exposición a riesgo biológico y los factores que contribuyeron al evento.

\section{Materiales y Métodos}

Tipo de estudio y muestra: se trató de un estudio descriptivo y retrospectivo, que consideró como muestra de estudio todos los reportes de accidentes ocurridos entre el 2014 y 2016 en los trabajadores de salud de una IPS de segundo nivel de complejidad ubicada en la ciudad de Palmira-Valle del Cauca.

Recolección de datos y variables de estudio: se diseñó una matriz de recolección de datos en hoja electrónica en Excel, con base en el protocolo de reporte de accidentes de trabajo de la institución 
fuente de la información. Se recolectaron 30 variables entre las que se calificó como dependiente el "accidente de trabajo por exposición a riesgo biológico" y como independientes: los datos sociodemográficos, laborales, temporales y del lugar donde ocurrió el AT, características de la lesión y de su ocurrencia, factores generadores presentes al momento del accidente y el manejo post-exposición.

Contexto del estudio: se trató de una institución prestadora de servicios de salud (IPS) con 320 trabajadores, de los cuales 27 eran médicos, 40 enfermeras profesionales, 122 tecnólogas en enfermería y 7 instrumentadores quirúrgicos. La institución cuenta con los servicios de Urgencias (19 camillas y 7 sillones), Imágenes Diagnósticas (Radiografías, Ecografías y Tomografías), Laboratorio Clínico, Hospitalización (48 camas), cuidado Intensivo e Intermedio del Adulto (12 camas), Sala de partos (puerperio 3 camas - trabajo de parto 4 camas) y Cirugía en los niveles I, II (3 quirófanos).

Manejo y análisis estadístico de los datos: todos los datos fueron gestionados en hoja electrónica Microsoft $\AA$ Excel y posteriormente exportados al paquete estadístico en el cual se llevó a cabo el análisis, SPSS vs 20 (IBM New York USA). A las variables cuantitativas se les calcularon las medidas de tendencia central y dispersión, a las cualitativas las proporciones y razones. Para evaluar la relación entre la variable resultado y las variables independientes durante el periodo de estudio, se utilizó la prueba de Chi2, test de Fischer y la prueba de Medianas, dependiendo del comportamiento normal o no de las variables y del número de datos en las casillas de las tablas de contingencia, considerando un valor de $\mathrm{p} \leq 0,05$ estadísticamente significativo.

Aspectos éticos: la investigación cumplió con los principios de la declaración de Helsinki y las normas descritas en la Resolución 8430 de 1993 del Ministerio de Salud de la República de Colombia. La propuesta de investigación fue avalada por el nivel directivo de la institución fuente de información y el Comité de Ética Humana de la Universidad LibreSeccional Cali.

\section{Resultados}

Un total de 37 casos fueron reportados durante el periodo de estudio, los cuales incluyeron 30 tecnólogas en enfermería. El 89 \% fueron mujeres, presentándose una razón de 8 mujeres por cada hombre. El promedio de edad fue 25,4 $\pm 3,9$ años. Más de la mitad (28/37) de los casos involucró a trabajadores que tenían 28 años o menos.

Considerando el tiempo laborado en la IPS, previo al reporte, el primer accidente laboral con exposición al riesgo biológico discriminado por cargo mostró que las enfermeras profesionales se demoraron aproximadamente 1,6 años antes de sufrir un evento y en general, 19 de los trabajadores, menos de un año. La proporción de trabajadores con menos de dos años de antigüedad (66,7\%) en la institución y con más de dos $(33,3 \%)$ que sufrieron un accidente laboral por exposición a factor de riesgo biológico, presentó diferencias estadísticamente significativas (Prueba de Medianas, Valor de $\mathrm{p}=0,004$ ). Por otra parte, con relación a las variables temporales se identificó accidente que ocurrió un accidente en fin de semana y dos entre semana dos (Prueba $\mathrm{Chi}^{2}$, Valor de $\mathrm{p}=0,010$ ). Además, el $64 \%$ de los accidentes biológicos sucedieron en horas de la mañana.

De los 37 casos incluidos en el estudio, 30 fueron con elementos punzantes. Por cada 10 accidentes biológicos se presentaron 8 que involucraron elementos punzantes. El área del cuerpo más afectada fueron las manos (34/37). En la revisión de los reportes del AT se encontró que 33 de los trabajadores no estaban usando materiales o equipos de seguridad al momento del accidente. En la tabla 1 se presenta un resumen de las variables que describen las características de la lesión y sus causas. 
ISSN-PRINT

1794-9831

E-ISSN 2322-7028

Vol. 15 No. 2

Jul - Dic 2018

Cúcuta, Colombia

Tabla 1. Características del accidente laboral con exposición al riesgo biológico en una IPS de segundo nivel. 2014-2016.

\begin{tabular}{llcc}
\hline \multicolumn{1}{c}{ Variables } & \multicolumn{1}{c}{ Categorías } & Casos & Porcentaje (\%) \\
\hline Tipo de lesión & Herida & 37 & 100 \\
\multirow{2}{*}{ Tipo de elemento involucrado } & Punzante & 30 & 81 \\
& Corto punzante & 1 & 3 \\
& Mordedura & 6 & 16 \\
\cline { 2 - 2 } Área del cuerpo & Manos & 34 & 92 \\
& Miembros superiores & 2 & 6 \\
& Miembros inferiores & 1 & 3 \\
Causa del accidente de trabajo & Manipulación de elementos punzantes (agujas) & 18 & 48,6 \\
& Durante la atención al paciente & 15 & 40,5 \\
\hline
\end{tabular}

Fuente: Datos del trabajo de investigación.

Los accidentes ocurrieron con mayor frecuencia en el servicio de urgencias (17/37). Al indagar por la actividad que estaba realizando el trabajador al momento del accidente discriminada por servicio, se encontró que predominaron las lesiones causadas por manipulación de agujas (9/18), por el proceso de atención al paciente $(53,3 \%)$ en el área de urgencias y por la atención al enfermo en el servicio de hospitalización $(33,3 \%)$ sin diferencias significativas (Prueba de $\mathrm{Chi}^{2}$, Valor de $\mathrm{p}=0,397$ ).
La media y mediana de pacientes atendidos por servicios en los horarios en los cuales ocurrieron los accidentes con exposición al riesgo biológico se presentan en la tabla 2. Se observaron diferencias estadísticamente significativas entre el promedio de eventos ocurridos en el servicio de urgencias con relación al resto (Prueba de medianas; valor de $\mathrm{p}=0,025$ ) y entre hospitalización y los otros servicios (Prueba de medianas; valor de $\mathrm{p}=0,007)$ así como entre urgencias y hospitalización (Prueba de medianas; valor de $\mathrm{p}=0,000$ ).

Tabla 2. Número de pacientes atendidos según servicios en las IPS fuente de información. 2014-2016.

\begin{tabular}{lcc}
\hline Servicio & $\begin{array}{c}\text { Media del } \mathbf{N}^{\circ} \text { de } \\
\text { pacientes atendidos }\end{array}$ & $\begin{array}{c}\text { Mediana del } \mathbf{N}^{\circ} \text { de } \\
\text { pacientes atendidos }\end{array}$ \\
\hline Urgencias & 127 & 133 \\
Hospitalización & 55 & 58 \\
Otros & 31 & 5 \\
\hline
\end{tabular}

Fuente: Datos del trabajo de investigación.

El $62,2 \%$ de los reportes del AT fueron hechos en menos de 48 horas y el resto más allá de las 48 horas. Este dato discriminado por servicio no mostró diferencias estadísticamente significativas (Test de Chi2, valor de $\mathrm{p}=0,626)$. Al evaluar el cumplimiento del esquema de vacunación se encontró que 31 del total de los trabajadores accidentados cumplían, 3 tenían solo una dosis y un trabajador dos dosis, otro asistencial no tenía ninguna dosis y en un caso no se encontró registro del dato.

En cuanto al cumplimiento del protocolo postexposición institucional para la toma de exámenes de laboratorio, solo a un caso se le aplicó el esquema completo, es decir, tanto al trabajador como a la fuente. En la tabla 3 se presenta un resumen de los análisis reportados en las historias de salud ocupacional. 
Tabla 3. Números de exámenes hechos a la fuente y al paciente de los casos incluidos en el estudio.2014-2016.

\begin{tabular}{|c|c|c|}
\hline EXAMEN & TRABAJADOR & FUENTE \\
\hline Serología & 29 & 16 \\
\hline VIH & 35 & 20 \\
\hline Anticuerpos para Hepatitis C & 25 & 16 \\
\hline Anticuerpos para Hepatitis B & 5 & NA* \\
\hline Antígenos de superficie para Hepatitis B & 33 & 20 \\
\hline
\end{tabular}

Vol. 15 No. 2

Jul - Dic 2018

Fuente: Datos del trabajo de investigación.

Con relación al seguimiento: al $62 \%$ se le hicieron exámenes al finalizar el primer mes; al $27 \%$ al tercer mes; $16 \%$ al sexto mes y a ninguno de los trabajadores accidentados se le practicaron los exámenes de seguimiento al cumplir el año. No se observaron diferencias estadísticamente significativas en esta variable según servicio (Test de Fisher, valor de $\mathrm{p}=0,781$ ) (Tabla 4).

Tabla 4. Seguimientos de exámenes al trabajador expuesto a factor de riesgo biológico. 2014-2016.

\begin{tabular}{lcccc}
\hline Exámenes & $\begin{array}{c}\text { Primer } \\
\text { mes }\end{array}$ & $\begin{array}{c}\text { Tercer } \\
\text { mes }\end{array}$ & $\begin{array}{c}\text { Sexto } \\
\text { mes }\end{array}$ & Año \\
\hline No & 11 & 24 & 31 & 34 \\
$\mathbf{S i}$ & 23 & 10 & 6 & 0 \\
Sin dato & 3 & 3 & 0 & 3 \\
\hline
\end{tabular}

Fuente: Datos del trabajo de investigación.

Al comparar el número de trabajadores que usaban los EPP con los que no los usaban al momento del AT se observó que existía una relación de 1 a 8 . No se presentaron diferencias estadísticamente significativas entre el grupo de trabajadores que habían recibido capacitación sobre temas de riesgo biológico, procedimientos a llevar a cabo en la postexposición, la importancia y buen uso de los EPP con relación a los que no fueron capacitados (Test de $\mathrm{Chi}^{2}$, valor de $\left.\mathrm{p}=0,397\right)$. Por otra parte, se comprobó que de los 9 trabajadores que recibieron capacitación sobre el correcto uso de los EPP y su importancia, 7 no estaban usando los EPP al momento del AT, lo que permite concluir que no hay asociación entre las dos variables (Test de Fisher, valor $\mathrm{p}=0,224$ ).

\section{Discusión}

Las características sociodemográficas de la población mostraron como prevalente a los tecnólogos y profesionales de enfermería, principalmente mujeres jóvenes que laboraban en los servicios de urgencias y hospitalización.

Resultados similares fueron reportados por Correa y Parada (16), Hassanpour et al. (17) así como por Gopar et al. (18), quienes expusieron que los trabajadores de la salud que predominan en más del $50 \%$ de los accidentes laborales con material biológico son mujeres jóvenes, tecnólogas en enfermería que se desempeñan en el servicio de urgencias. Esta situación se prevé, ya que son los trabajadores asistenciales que más prevalecen en el ambiente hospitalario. Es un personal cuyas actividades están relacionadas con la asistencia directa al paciente y el empleo de una gran cantidad de procedimientos que involucran elementos punzantes y agujas. Estos resultados contrastaron con los de Ruas et al. (19) al afirmar que la unidad de hospitalización es el servicio con mayor prevalencia de AT. Por otra parte, fueron similares a los reportados por Ottobelli et al. (20) quienes indican que son los tecnólogos en enfermería los que se 
ISSN-PRINT

1794-9831

E-ISSN 2322-7028

Vol. 15 No. 2

Jul - Dic 2018

Cúcuta, Colombia imponen como población vulnerable, siendo jóvenes del sexo femenino.

La sobrecarga laboral, es otro factor asociado muy frecuente en este tipo de eventos, principalmente en países de ingresos bajos y medios $(21,22)$. Esta variable fue significativa en los resultados de este estudio. El servicio donde se desempeñaban los trabajadores que reportaron AT con material biológico se relacionó con el servicio en donde se atendieron el mayor número de pacientes.

Otro factor presente fue la antigüedad en la IPS. Esta característica puede estar asociada a la poca experiencia laboral dado que un alto porcentaje de la población estudiada tenía menos de 28 años. Resultados semejantes fueron reportados por Machado et al (23), al demostrar que los asistenciales con menos experiencia laboral fueron los que más accidentes con exposición a riesgo biológico reportaron. Si bien en la formación académica de los trabajadores de la salud se incluye el componente del riesgo biológico, la prevención y manejo de estos, pareciera que se está adquiriendo con la experiencia laboral y la capacitación que reciben en sus sitios de trabajo. En este sentido, los reportes de varios estudios muestran que existe una relación entre antigüedad laboral o experiencia laboral y mayor conocimiento en accidentes laborales por exposición al riesgo biológico (23-25). Estos resultados son consistentes con los de este estudio, en donde se identificó que los trabajadores con mayor antigüedad fueron los que menos se vieron involucrados en reportes de AT con material biológico. En contraste, otros investigadores han encontrado que la mayoría de los accidentes ocurren en profesionales de enfermería con más de 10 años de experiencia asistencial (26).

Por otra parte, en el presente estudio se comprobó que el turno en que más se notificaron accidentes correspondió al matutino seguido por el nocturno, coincidiendo con Clemente et al. (27) y Correa y Parada (16), para quienes esto puede corresponder a que son los turnos donde se realizan la mayor cantidad de procedimientos de atención como: canalizaciones, curaciones, baño de pacientes, tomas de muestras de sangre para controles y administración de medicamentos, entre otros. Adicional a estos hallazgos, en esta investigación se evidenció que fueron los turnos en donde se atendieron el mayor número de pacientes.

El tipo de AT con material biológico que predominó en más del $70 \%$ de los casos en estudio fue el percutáneo (por punción con agujas). Las causas más frecuentes fueron el movimiento del paciente o paso de otro usuario cuando se estaba realizando algún procedimiento que involucraba un elemento punzante, y en menor cantidad durante el descarte y el re-encapsulamiento de las agujas. Estos hallazgos fueron semejantes a los reportados por Tipayamongkholgul et al. (14) y Machado et al. (23). Cabe resaltar que, para este estudio la proporción de trabajadores accidentados por reencapsular agujas fue significativamente baja en contraste con los estudios de Tadesse y Tadesse (2) y Jahangiri et al. (22), en los que esta causa se presentó en el $40 \%$ de los casos.

La oferta de programas de capacitación en el tema del riesgo biológico y el procedimiento a seguir en caso de ocurrencia de AT está asociada con la mitigación del riesgo $(22,28,29)$. Los resultados del presente trabajo encontraron una baja proporción de personal accidentado capacitado en el tema. En contraste, Muñoz et al. (30) mostraron que más del $70 \%$ de su población refirió haber recibido capacitaciones en los últimos dos años. En contraste, los estudios de Hadaway (31) y Gourni et al. (32), revelaron una baja proporción de trabajadores capacitados en el tema de "que hacer" al momento del accidente, por lo que son necesarios los programas de capacitación continua.

El correcto uso de los EPP y su importancia para la prevención de los AT con material biológico son temas importantes a tener en cuenta en los programas de capacitación, inducción y reinducción para la mitigación del riesgo. Ndejjo et al. (33), expusieron que la capacitación permanente sobre la importancia del uso de los EPP en el proceso asistencial, se asoció con una exposición reducida a los riesgos biológicos. Los hallazgos de Ndejjo et al. (33) apoyan la evidencia de Hayden et al. (36), quienes también indicaron que el uso de EPP redujo la adquisición de enfermedades en los trabajadores asistenciales. De hecho, durante mucho tiempo se ha reconocido que los EPP son una importante medida de control del riesgo biológico en la asistencia sanitaria $(22,38)$, lo que debe subrayarse para minimizar la exposición a los riesgos profesionales en ambientes hospitalarios. El no uso de los elementos de protección personal (EPP), por parte del personal capacitado y el no capacitado en el tema fue un factor presente en los resultados de este estudio. Esto refleja que más allá del desconocimiento como posible causa del evento está, la baja percepción del riesgo. De igual forma se puede concluir como factor desencadenante 
el no contar con los suficientes insumos para el proceso asistencial, dato que no fue posible recolectar y que según los reportes de varios estudios ha sido una variable identificada (33-36). Ahora, no solo se debe considerar la disponibilidad de los EPP dado que en un estudio reciente llevado a cabo por Trindade et al. (37), encontró que la calidad de los guantes también tiene un papel importante en la exposición al material biológico. En su estudio casi el $50 \%$ (135/300) de estos EPP usados tanto en procedimientos de limpieza y esterilización de material hospitalario como en salas de cirugía estaban perforados

El manejo correcto y oportuno post-exposición también contribuye a la mitigación del riesgo. Un estudio llevado a cabo en Colombia entre los años 2012 y 2013 (23), mostró que algo más del $70 \%$ de los accidentes fueron reportados de manera oportuna, por lo cual el evento no terminó en efectos colaterales graves para el trabajador. Los resultados de este estudio, en un alto porcentaje, fueron semejantes. Por otra parte, si bien a un alto porcentaje de los pacientes que fueron fuente de origen del fluido biológico con el cual tuvo contacto el trabajador asistencial, se les practicaron estudios para evaluar su infectividad con respecto a algunos de los agentes infecciosos de importancia en este campo, no se cumplió en su totalidad el protocolo de exámenes de control o seguimiento en los trabajadores accidentados. Situación similar fue identificada por Machado et al. (23) en un estudio realizado en Colombia. El no cumplimiento de los protocolos de vigilancia conlleva a la insatisfacción de los trabajadores asistenciales que reportan sobre estos eventos y con el tiempo contribuyen al sub-registro del mismo (39).

Por último, se puede deducir que los resultados de este estudio muestran que las variaciones en la prevalencia de los diferentes AT con material biológico y sus factores asociados difieren según: el servicio, el profesional asistencial involucrado, la disparidad en el número de trabajadores de salud en las diferentes áreas, el número de pacientes atendidos, el tipo de elementos usados y los procedimientos practicados durante la asistencia al paciente. Sin embargo, está claro que no se pueden hacer extensivos estos resultados a otras organizaciones puesto que como lo expresaron Tadesse y Tadesse (2) y Tayar et al. (39), hay variaciones en la prevalencia de este tipo de eventos según la organización y otros factores que no fueron considerados en este estudio. De aquí que sea necesario indagar por los datos, que según estudios internacionales son covariables de las variables analizadas en esta investigación, como son el nivel de estrés del trabajador (40), el clima organizacional (41), el nivel salarial, la inversión presupuestal para los programas de seguridad y salud laboral (42) y la gestión del riesgo biológico (39). Estas variables en conjunto podrían ayudar a definir un poco más los contextos organizacionales que contribuyen a la ocurrencia de los AT.

\section{Conclusiones}

- El perfil de los accidentes de trabajo con exposición a riesgo biológico englobó a los servicios de mayor demanda asistencial, en el turno matutino, personal joven, de formación tecnológica, con poca antigüedad en la empresa.

- Los resultados de este estudio mostraron que el tipo de elemento vulnerante, el sitio de la lesión y el no uso de los EPP contribuyen en la definición del perfil epidemiológico de las lesiones.

- Los factores que contribuyeron con los AT están presentes en las actividades en donde se involucra la manipulación de elementos punzantes cuando se asiste al paciente, sumado a movimientos bruscos de este. Así mismo se identificó que el riesgo se incrementa cuando no se lleva a cabo una buena gestión en la postexposición.

- Varias de las causas asociadas al evento hacen parte del grupo de factores modificables, lo que estaría indicando que los programas de capacitación continua y sensibilización con su debido seguimiento y apoyo a la gestión del programa de salud y seguridad laboral por parte de la organización, podrían contribuir a disminuir los índices de los AT con exposición a material biológico.

- Para finalizar, toda la revisión bibliográfica hecha permite concluir que se necesitan investigaciones que cuantifiquen la carga laboral, el estrés, el clima organizacional y el factor comportamental en los trabajadores de la salud, en la ocurrencia de estos eventos así como los impactos a largo plazo, para lo cual es necesario contar con sistemas de información de alta calidad que faciliten los estudios sobre este tema. Para esto se requiere fortalecer y dar sostenibilidad a los sistemas de información. 
ISSN-PRINT

1794-9831

E-ISSN 2322-7028

Vol. 15 No. 2

Jul - Dic 2018

Cúcuta, Colombia

\section{Agradecimientos}

Los investigadores agradecen la información suministrada por las Directivas de la institución y la Coordinación del Programa de Seguridad y Salud Laboral.

\section{Conflictos De Interés}

Los investigadores declaran que no existe ningún tipo de conflicto de intereses con respeto a los resultados de esta investigación.

\section{Referencias Bibliográficas}

1. Cooke CE, Stephens JM. Clinical, economic, and humanistic burden of needlestick injuries in healthcare workers. Medical Devices: Evidence and Research. [Internet]. 2017 [consultado el 28 de Abril de 2018]; 10:225-235. Disponible en: https://www.dovepress.com/clinical-economic-and-humanistic-burden-ofneedlestick-injuries-in-hea-peer-reviewed-fulltext-article-MDER

2. Tadesse M, Tadesse T. Epidemiology of needlestick injuries among health-care workers in Awassa City, Southern Ethiopia. Trop Doct. [Internet]. 2010 [consultado el 13 de Marzo de 2018]; 40(2): 111-13. Disponible en: http://journals.sagepub.com/doi/abs/10.1258/td.2009.090191

3. Wilburn SQ, Eijkemans G. Preventing Needlestick Injuries among Healthcare Workers: A WHO-ICN Collaboration. Int j occup environ health. [Internet]. 2004 [consultado el 10 de Noviembre de 2016]; 10:451-456. Disponible en: http://www.tandfonline.com/doi/abs/10.1179/oeh.2004.10.4.451

4. Salles Rodrigues P, Lopes de Sousa AF, da Silva Magro MC, de Andrade D, de Souza Hermann PR. Occupational accidents among nursing professionals working in critical units of an emergency service. Esc. Anna Nery [Internet]. 2017 [consultado el 29 de Abril de 2018]; 21(2):1-6. Disponible en http://www.scielo. br/pdf/ean/v21n2/1414-8145-ean-21-02-e20170040.pdf.

5. Masoumi Asl H, Rahbar M, Soltani A, Pezeshki Z, Khanaliha K, Kolifarhood G. Epidemiology of Needlestick Injuries Among Healthcare Workers in Tehran, Iran: A Cross-Sectional Study. Archives of Clinical Infectious Diseases [Internet]. 2016 [consultado el 4 de Julio de 2017]; 12(2):1-7. Disponible en: https://www.researchgate.net/publication/310050211_Epidemiology_of_Needlestick_Injuries Among_Healthcare_Workers_in_Tehran_Iran_A_Cross-Sectional_Study

6. Cho E, Lee H, Choi M, Park SH, Yoo IY, Aiken LH. Factors associated with needlestick and sharp injuries among hospital nurses: A cross-sectional questionnaire survey. International Journal of Nursing Studies [Internet]. 2013 [consultado el 3 de Mayo de 2017]; 50(8):1025-1032. Disponible en: http:// www.sciencedirect.com/science/article/pii/S0020748912002489

7. Montufar FE, Madrid C, Villa JP, Díaz LM, Vega J, Vélez JD et al. Accidentes ocupacionales de riesgo biológico en Antioquia, Colombia. Enero de 2010 a diciembre de 2011. Infectio. [Internet]. 2014 [consultado el 28 de Abril de 2018]; 18(3):79-85. Disponible en http://www.elsevier.es/es-revistainfectio-351-articulo-accidentes-ocupacionales-riesgo-biologico-antioquia-S0123939214000265

8. López AM, Rúa CM, Montoya FA. Factores personales y laborales del personal asistencial relacionados con la ocurrencia de accidentes por riesgo biológico de la ESE Popayán 2008-2010. [Tesis] Popayán, Colombia. Universidad Libre. Facultad de ciencias de salud; 2010.

9. Arenas Sánchez A, Pinzón Amado A. Riesgo biológico en el personal de enfermería: una revisión práctica. Revista CUIDARTE [Internet]. 2011 [consultado el 25 de abril de 2018]; 2(1):216-224. Disponible en http:// www.redalyc.org/pdf/3595/359533178018.pdf

10. Montufar FE, Villa JP, Madrid CA, Díaz LM, Vega J, et al. Infección por VIH posterior a exposición ocupacional de riesgo biológico en trabajadores de la salud. Rev. Infectio. [Internet]. 2015 [consultado el 5 de Noviembre de 2017]; 19(1):31-34. Disponible en http://www.sciencedirect.com/science/article/ pii/S0123939214000277

11. Arenas Sanchez A, Pinzón Amado A. Riesgo biológico en el personal de enfermería: una revisión práctica. Rev Cuid. [Internet]. 2011 [consultado el 17 de Marzo de 2017]; 2(1):216-24. Disponible en: http:/www. redalyc.org/html/3595/359533178018/ 
12. Felix AMS, Victor ES, Malaguti SET, Gir E. Fatores individuais, laborais e organizacionais associados à adesão às precauções-padrão. J Infect Control. 2013; 2(2):1-5.

13. Duarte VM, Palucci MMH, Miyeko H, Richart-Martínez M. Occurrence of occupational accidents involving potentially contaminated biological material among nurses. Acta paul. enferm. 2014; 27(3):280-286.

14. Tipayamongkholgul M, Luksamijarulkul P, Mawn B, Kongtip P, Woskie S. Occupational Hazards in the Thai Healthcare Sector. New solutions : a journal of environmental and occupational health policy : NS. 2016; 26(1):83-102.

15. Azadi A, Anoosheh M, Delpisheh A. Frequency and barriers of underreported needlestick injuries amongst Iranian nurses, a questionnaire survey. J Clin Nurs. 2011; 20(3-4):488-93.

16. Correa DX, Parada DA. Accidentes con riesgo biológico en una población afiliada a una ARL, 2014, Colombia. J. Chem. Inf. Model. 2014; 53(9):1689-1699.

17. Hassanpour A, Mohammadi N, Nikbakht A. Redesigning Orem's Self-care theory for patients with chronic hepatitis Indian J Palliat Care. 2016; 22(4):395-401.

18. Gopar Nieto R, Juárez Pérez CA, Cabello López A, Haro García LC, Aguilar Madrid G. Overview of sharps injuries among health-care workers. Rev Med Inst Mex Seguro Soc. [Internet]. 2015 [consultado el 25 de Abril de 2018]; 53(3):356-361. Disponible en http://revistamedica.imss.gob.mx/ editorial/index.php/revista_medica/article/view/40/778

19. Ruas EFG, Santos L, Barbosa DA, Belasco AGS, Bettencourt ARC. Acidentes ocupacionais com materiais perfurocortantes em hospitais de Montes Claros-MG. REME Rev. Min. Enferm. 2012; 16 (3):437-43.

20. Ottobelli C, Cezar Vaz MR, dos Santos CargninMC, ArgentaC, ZanattaRG. Acidentes de trabalho com perfurocortantes em unidade de centro cirúrgico na Região Sul do Brasil. O Mundo da Saúde, São Paulo. [Internet]. 2015 [consultado el 28 de Abril de 2018]; 39(1):113-118. Disponible en http://bvsms.saude. gov.br/bvs/periodicos/mundo_saude artigos/acidentes trabalho \%20perfurocortantes.pdf.

21. Rodrigues PS, Sousa AF, Magro MC, Andrade D, Hermann PR. Occupational accidents among nursing professionals working in critical units of an emergency service. Esc. Anna Nery. 2017; 21(2):1-6.

22. Jahangiri M, Rostamabadi A, Hoboubi N, Tadayon N, Soleimani A. Needle Stick Injuries and their Related Safety Measures among Nurses in a University Hospital, Shiraz, Iran. Safety and Health at Work. 2016; 7(1):72-77.

23. Machado JE, Cardona BE, González RL. Adherencia al protocolo de manejo de accidente por riesgo biológico en una administradora de riesgos laborales de Colombia, 2012-2013. Revista Ciencia y Trabajo. 2014. 16(50):103-110.

24. Olufemi OA, Ayobami EA, Titilayo FA, Mathew KE, Abiodun TA, Bukola FP. Knowledge, attitudes and perceptions of occupational hazards and safety practices in Nigerian healthcare workers. BMC Res Notes. [Internet]. 2016 [consultado el 17 de Marzo de 2017]; 9:71. Disponible en https://www. ncbi.nlm.nih.gov/pmc/articles/PMC4744628/pdf/13104_2016_Article 1880.pdf

25. Echeverri MP, Salcedo-Cifuentes M. Conocimientos y Actitudes en la Aplicación de Normas de Bioseguridad del Personal del Servicio de Enfermería. Revista Colombiana de Salud Ocupacional. 2014; 4(1):15-21.

26. Pimenta FR, Ferreira MD, Gir E, Hayashida M, Canini SRMS. Care and specialized clinical follow-up of nursing professionals who have been victims of accidents with biological material. Rev Esc Enferm USP [Internet]. 2013; 47(1):198-204.

27. Clemente M, Guzmán CK, Martínez M, Álvarez MC, Sagües MJ. Accidentes percutáneos con riesgo biológico, producidos por dispositivos de seguridad en la Comunidad de Madrid. Med Segur Trab. 2012; 58(227):82-97.

28. Rim K-T, Lim C-H. Biologically Hazardous Agents at Work and Efforts to Protect Workers' Health: A Review of Recent Reports. Safety and Health at Work. 2014; 5(2):43-52.

29. Dickmann P, Bhatiasevi A, Chaib F, Baggio O, Banluta C, et al. Biological Risks to Public Health: Lessons 
ISSN-PRINT

1794-9831

E-ISSN 2322-7028

Vol. 15 No. 2

Jul - Dic 2018

Cúcuta, Colombia

from an International Conference to Inform the Development of National Risk Communication Strategies: Report of an international conference on risk communication strategies before, during, and after public health emergencies, Rabat, Morocco, October 22-23, 2015. Health Security. 2016; 14(6):433-440.

30. Muñoz R, Briceño $M$, Donoso $D$, Tetamantti $D$. Accidentes laborales con exposición a material biológico y grupo más sensible a los mismos (ALEMB), hospitales "Luis Vernaza", maternidad "Enrique C. Sotomayor", maternidad "Mariana de Jesús", del niño "Francisco de Ycaza Bustamante" y "Abel Gilbert Ponton" de Guayaquil. Rev. Medicina. 2010; 16(1):18-24.

31. Hadaway L. Needlestick injuries, short peripheral catheters, and health care worker risks J Infus Nurs. 2012; 35:164-178.

32. Gourni P, Polikandrioti M, Vasilopoulos G, Mpaltzi E, Gourni M. Occupational exposure to blood and body fluids of nurses at Emergency department Health Sci J. 2012; 6:60-68.

33. Ndejjo R, Musinguzi G, Yu X, Buregyeya E, Musoke D, Wang JSH, et al. Occupational Health Hazards among Healthcare Workers in Kampala, Uganda. Journal of Environmental and Public Health. 2015.

34. Adriane Corrêa J, Palucci Marziale ME, Benedita dos Santos C, Spadotti Dantas RA, Ko Nai Y. Assessment of adherence to post-exposure conducts among health workers: translation and cultural adaptation of an instrument. Texto contexto - enferm. 2015; 24 (3):670-679.

35. Romana C, Corrao N, Mazzotta A, La Torre G, De Giusti M. Biological Risk and Occupational Health. Industrial Health. 2012; 50:326-337.

36. Hayden MK, Blom DW, Lyle EA, Moore CG, Weinstein RA. Risk of hand or glove contamination after contact with patients colonized with vancomycin-resistant Enterococcus or the colonized patients' environment. Infection Control and Hospital Epidemiology. 2008; 29(2):149-154.

37. Trindade Pires JA, Rutyelle da Serra J, Veiga Tipple AF. Índice de perfuração de luvas de procedimento/ cirúrgica utilizadas por trabalhadores do expurgo de um centro de material e esterilização. Texto contexto - enferm. [Internet]. 2016 [consultado el 28 de Abril de 2018]; 25(2): e1410015. Disponible en http://www. scielo.br/scielo.php?script $=$ sci arttext\&pid $=$ S0104-07072016000200319\&lng=en\&nrm $=$ iso\&tlng $=p t$

38. Resende A, Veríssimo AG, Rosendo da Silva RA, de Moura AK, Queiroz AC. Work accidents with exposure to biological material in nursing in emergency rooms. J Nurs UFPE. 2014; 8(4):910-8.

39. Tayar L, Gir E, Pazin-Filho A, Hayashida M, Marin SR. Under-Reporting of Accidents Involving Biological Material by Nursing Professionals at a Brazilian Emergency Hospital, International Journal of Occupational Safety and Ergonomics. 2013; 19(4):623-629.

40. Jetha A, Kernan L, Kurowski A. Conceptualizing the dynamics of workplace stress: a systems-based study of nursing aides. BMC Health Serv Res. 2017; 17:12.

41. d'Ettorre G. Job stress and needlestick injuries: which targets for organizational interventions?. Occupational Medicine. [Internet]. 2016 [consultado el 24 de Abril de 2018]; 66(8):678-680. Disponible en https://www.ncbi.nlm.nih.gov/pubmed/27481860

42. Santos SVM, Macedo FRM, Silva LA, Resck ZMR, Nogueira DA, Terra FS. Work accidents and selfesteem of nursing professional in hospital settings. Rev. Latino-Am. Enfermagem [Internet]. 2017 [consultado el 13 de Marzo de 2018]; 25(e2872):1-8. Disponible en http://www.scielo.br/pdf/rlae/v25/ es 0104-1169-rlae-25-2872.pdf

43. Duarte Valim M, Palucci Marziale MH, Hayashida M, Richart Martínez M. Occurrence of occupational accidents involving potentially contaminated biological material among nurses. Acta paul. enferm. $2014 ; 27(3): 280-286$. 